\title{
IMPLANTAÇÃO DO PAGAMENTO INSTANTÂNEO NO MERCADO BRASILEIRO DE SEGUROS
}

IMPLEMENTATION OF INSTANT PAYMENT IN THE BRAZILIAN INSURANCE MARKET

\section{Fernando Igor Ferreira Costa}

Mestre em Administração do Desenvolvimento de Negócios pela Universidade Presbiteriana Mackenzie (UPM).

Gerente de produtos.

E-mail:fernandicosta@hotmail.com

Alexandre Cappellozza

Doutor em Administração pela EAESP-FGV.

Coordenador do Programa de Pós-Graduação em Administração do Desenvolvimento de Negócios da UPM. 


\section{Gustavo Hermínio Salati Marcondes de Moraes}

Professor livre-docente na Universidade Estadual de Campinas (Unicamp). Coordenador do Programa de Pós-Graduação em Administração da Unicamp. E-mail:salati@unicamp.br

\section{RESUMO}

A indústria de cartões passa por evoluções que se intensificaram nos últimos anos, como a maior regulação do Banco Central do Brasil, por exemplo, que determinou a quebra do monopólio da adquirência das bandeiras de cartões Visa, Mastercard, Elo, American Express e Hipercard. Contudo, as maiores mudanças estão relacionadas com inovações, que permitiram novos produtos e serviços e contribuíram para novas experiências de pagamento e formas de captura de transações. Este trabalho apresenta a implantação do pagamento instantâneo por uma adquirente para distribuição de fundos das seguradoras para os segurados. Este trabalho adota o método de design science sob o modelo de problem-solving, com técnicas mistas de análise nas etapas de diagnóstico e avaliação com uma abordagem quantitativa, pela aplicação de um questionário em 124 respondentes, e outra qualitativa, com entrevistas com gestores de seguradoras e gestores comerciais da adquirente. Como benefício da solução, destacam-se a disponibilidade, a velocidade do envio de informações, a segurança da transação e a conveniência. Além disso, o trabalho mostra uma solução aplicada em um mercado pouco explorado pelas organizações desse segmento com a apresentação da jornada de desenvolvimento e fatores de sucesso para o desenvolvimento dessa inovação tecnológica.

\section{PALAVRAS-CHAVE}

Adquirente. Pagamento instantâneo. Distribuição de fundos. Seguradoras.

\section{ABSTRACT}

The card industry is going through significant evolutions, which have intensified in recent years, and it is possible to highlight the greater regulation of the Central Bank of Brazil, which determined the break of the acquiring monopoly of Visa, Mastercard, Elo, American Express and Hipercard. However, the biggest changes are related to innovations, which not only enabled new products and services, but also 
contributed to an easy payment experience, such as new payment experiences and transaction capture ways. This paper presents the implementation of the availability of instant payment by an acquirer for distribution of funds from insurers to policyholders. This manuscript adopts the design science method under the problem-solving model with mixed analysis techniques in the stages of diagnosis and assessment with a quantitative approach by applying a questionnaire to 124 respondents and qualitative with interviews with insurance managers and commercial managers of the acquirer. As a benefit of the solution, the availability, speed of sending information, transaction security and convenience stand out. The work shows a solution applied in a market little explored by organizations in this segment with the presentation of the development journey and success factors for the development of this technological innovation.

\section{KEYWORDS}

Acquirer. Instant payment. Fund distribution. Insurers. 


\section{INTRODUÇÃO}

Sabe-se que as inovações tecnológicas mudam a forma de comunicação e aquisição de produtos e serviços e isso inclui os meios de pagamento que precisam ser transparentes, fáceis, sem causar atrito no momento da compra (Toucinho, 2020). Assim, apesar dos restritos espaços de atuação para bancos de pequeno e médio porte no Brasil, com as novas tecnologias, possíveis disrupções na arena de competição bancária devem ocorrer (Lima, 2016). Os bancos e as autoridades reguladoras estão incentivando a migração dos pagamentos para a esfera digital, o que pode levar à redução dos custos de transação e, consequentemente, à redução dos custos operacionais (Stankic, Aleksic, \& Jakovljevic, 2019). No cenário de transformação digital do pagamento é possível identificar a facilidade das compras virtuais, o crescimento exponencial das carteiras digitais que permitem a transferências do dinheiro de forma rápida e segura com uso aplicado em diversos ramos de atividade.

Segundo dados do Banco Central do Brasil (BCB) (2020), observou-se o crescimento de cartões em uso no Brasil: em 2019, o percentual de aumento dos cartões de crédito ativos e cartões de débito ativos foi igual a 18\% e 14\%, respectivamente, na comparação com o ano de 2018. Além disso, ocorreu crescimento de 35\% no número de transações com cartão de crédito e $20 \%$ com cartão de débito. Esse crescimento de cartões ativos e das transações em meios eletrônicos pode ter promovido novas empresas para o setor de pagamentos brasileiro, o que aumentou a competição com empresas locais e globais (Braido, Klein, \& Papaleo, 2021).

Nos últimos anos, as tecnologias de informação estão mudando o comportamento dos consumidores, seja por fatores de pressão, que afastam os clientes dos serviços atuais, fatores de atração, que atraem os clientes para novas alternativas, ou por fatores de amarração, que impulsionam a mudança (Fan, Zhang, Rai, \& Du, 2021). No mercado de seguros, essa mudança comportamental é perceptível na forma como os clientes das seguradoras contratam e usam os serviços: a interface e o contato direto com o consumidor ganham espaço em detrimento da intermediação do corretor. Além do relacionamento direto, as soluções e os produtos tornaram-se mais simples para facilitar a aquisição e o uso dos diversos tipos de seguros (Lyskawa, Kedra, Klapkiv, \& Klapkiv, 2019). 
Nesse sentido, o mercado de seguros é um dos ramos de atividade que podem se beneficiar das inovações tecnológicas aplicadas ao pagamento, além de se tratar de um segmento em crescimento exponencial: segundo dados da Confederação Nacional das Empresas de Seguros Gerais, Previdência Privada e Visa, Saúde Suplementar e Capitalização (CNSEG) (2020), o setor arrecadou R 270 bilhões em receita com o crescimento de $8 \%$ em 2019. E é nesse contexto que as empresas se preparam para viabilizar a evolução dos meios de pagamento: a disponibilização do pagamento instantâneo com transferências e aquisição de produto e serviços por meio de transações de retirada e envio do dinheiro em segundos, disponível 24 horas por dia, sete dias por semana, sem as restrições dos métodos atuais, o que deve beneficiar a experiência do portador e estabelecimentos comerciais mais simples (Alfonso, Tombini, \& Zampolli, 2020).

A partir da disponibilidade do pagamento instantâneo no Brasil, este trabalho tem o objetivo de apresentar o aproveitamento dessa forma de pagamento por uma empresa adquirente do setor de meios de pagamento e o uso da tecnologia para envio de dinheiro das seguradoras para os segurados.

Este trabalho adota o método de design science research sob o modelo de problem-solving proposto por Van Aken, Berends e Van der Bij (2012), também detalhado em Marcondes, Miguel, Franklin e Perez (2017), que se baseia em cinco etapas para o desenvolvimento de soluções de problemas organizacionais: definição do problema ou oportunidade; diagnóstico e análise; projeto da solução; intervenção e aprendizagem; e avaliação. Há uma seção dedicada a cada um desses itens e, na sequência, as considerações finais.

\section{DEFINIÇÃO DA OPORTUNIDADE}

\section{mercado de meios de pagamento}

Segundo balanço divulgado pela Associação Brasileira das Empresas de Cartões de Crédito e Serviços (Abecs) (2021), o uso de cartões como meio de pagamento evoluiu no Brasil: movimentaram-se dois trilhões de reais em 2020 com cartões de crédito, débito e pré-pago, o que corresponde a um crescimento de $8,2 \%$ na comparação com o ano anterior. 
Além disso, contabilizaram-se R\$23,3 bilhões de pagamentos com cartões durante o ano, um volume 3,6\% maior que o observado no ano de 2019.

Destaca-se a maior adesão dos consumidores ao comércio on-line em decorrência da pandemia e da consequente necessidade de isolamento em 2020. Nesse sentido, notou-se um aumento de $32,2 \%$ nesse tipo de transação, o que representa um movimento de R 435 bilhões no uso de cartões na internet e outros tipos de transações sem a presença dos usuários no estabelecimento comercial.

O mercado - antes concentrado em dois concorrentes principais, respectivamente Visanet, atual Cielo, e Redecard, atualmente denominada Rede - ampliou-se com a determinação da quebra do monopólio da aceitação das bandeiras Visa e Mastercard pelo Banco Central do Brasil (BCB) em 2010, o que permitiu a criação e a expansão de novos entrantes, como Stone, Getnet e PagSeguro. Mesmo com o surgimento de novas empresas nos últimos anos, o mercado de meios de pagamento continua concentrado em poucas empresas, principalmente nas duas empresas incumbentes, Cielo e Rede, respectivamente, que juntas representam aproximadamente $60 \%$ do volume financeiro no terceiro trimestre de 2020.

No entanto, a concentração do market share das duas empresas dominantes não significa necessariamente lucro. Por exemplo, a Cielo tem apresentado queda em seu lucro nos últimos anos: no primeiro trimestre de 2020 somou R \$ 166,8 milhões, que é uma queda de 69,4\% em relação ao mesmo período de 2019 (Cielo, 2021). Desse modo, há uma motivação para as empresas constantemente descobrirem e implementarem estratégias que gerem valor a seus clientes, a necessidade de desenvolver a orientação para o mercado que pode maximizar os lucros (Jaworski \& Kohli, 1993).

As empresas da indústria de cartões precisam identificar novas opções de valor para os clientes, novas fontes de receita, estratégia de preço, de comunicação ou de produto para sobreviverem à nova dinâmica de mercado. A arena de competição deixou de ser restrita para poucos participantes, algo local, e passou a ser global. O investimento em tecnologia deixa de ser um diferencial e passa a ser uma obrigatoriedade no plano estratégico das instituições (Alfonso, Tombini, \& Zampolli, 2020). Recentemente, uma inovação do sistema financeiro teve destaque na mídia e referiu-se ao pagamento instantâneo com 
a movimentação de valores entre usuários e empresas de forma imediata sob o nome de Pix (Oxford Analytica, 2020).

\section{Modelo de pagamento instantâneo no Brasil}

A tecnologia divulgada em fevereiro de 2020 pelo BCB e lançada em novembro do mesmo ano sob a denominação Pix tem como objetivos principais: o aumento da disponibilidade de serviços financeiros para os usuários, a disponibilidade 24 horas por dia, sete dias da semana, e o aumento da conveniência com objetivo de criar uma experiência fácil de pagamento para os seus usuários (BCB, 2020).

A implantação do sistema pelas instituições de pagamento e fintechs permite transferências em dias não úteis e fora do horário comercial, sem custo para os usuários e/ou portadores de cartão, sendo um diferencial e motivo de migração dos modelos de transferências atuais, que cobram por transação (Campos, 2021). Além disso, a proposta da nova modalidade é que seja mais veloz que os métodos atuais e que a transação se concretize em até dez segundos com segurança, o que inclui a rede do Sistema Financeiro Nacional e o uso de diferentes métodos de autenticação do usuário, segundo dados do BCB (2020).

Outra alternativa para viabilizar o pagamento instantâneo é o uso de sistemas das bandeiras de cartões, como Visa, Mastercard e Elo, que conectam os players da cadeia, assim como no modelo de transações de compra atual, porém permitindo transações em tempo real. As bandeiras Visa e Mastercard, inclusive, já têm o modelo de pagamento instantâneo implantado em outros países com as marcas Visa Direct e Mastercard Send, respectivamente.

O modelo de pagamento instantâneo do BCB ultrapassou R 159 milhões de chaves cadastradas em janeiro de 2021 (Sgarioni, 2021, 17 de fevereiro). Esse cadastro é o primeiro passo para realização de transferências instantâneas e pode ser o cadastro de pessoas físicas (CPF) ou cadastro nacional da pessoa jurídica (CNPJ), e-mail, número de celular ou um código definido pelo portador. Já a quantidade mensal de transações foi de 200 milhões em janeiro de 2021 com um movimento de 160 bilhões de reais contra 144 milhões de transações em dezembro de 2020 e podem ser consideradas significativas, pois essa modalidade de pagamento foi lançada em novembro de 2020 (Campos, 2021). 
Embora o pagamento instantâneo já seja uma realidade no Brasil, o impacto que a tecnologia vai gerar no mercado ainda não é conhecido e, por isso, as empresas e os consumidores podem aprender com as experiências dos países que implementaram esse modelo de pagamento antes, como é o caso da China, da Índia e do Reino Unido (Demirgüç-Kunt, Klapper, Singer, Ansar \& Hess, 2018). Lançado em 2016, o sistema implantado na Índia é o líder mundial no número de pagamentos instantâneos com mais de 100 milhões de usuários ativos e aproximadamente 41 milhões de processamentos por dia (Conductor, 2020). Já a China, pioneira em pagamentos instantâneos, gerenciou 29 trilhões de dólares de transações digitais em 2017 de acordo com relatório do Banco Mundial (2017) (Demirgüç-Kunt et al., 2018).

\section{A empresa de adquirência e a oportunidade}

A empresa do presente trabalho, cujo nome será omitido por questões de sigilo, é responsável pela intermediação entre o estabelecimento comercial e o portador, em conjunto com os bancos emissores e as bandeiras de cartão, denominada adquirente. Suas atividades incluem o credenciamento, a transmissão, o processamento e a liquidação financeira das transações. A empresa transaciona mais de 20 bandeiras nacionais e outras internacionais de cartão de crédito e débito e tem capacidade tecnológica de gerar seis mil vendas por segundo e monitorar $100 \%$ das vendas, 24 horas por dia em todos os dias do ano.

Fundada na década de 1990 e sediada no estado de São Paulo, a adquirente está presente em mais de 5.500 municípios, com parceria ou participação em outras empresas do setor. Nos últimos anos, a empresa tem demonstrado a sua capacidade de inovação, como o lançamento da máquina de cartão com acesso às plataformas iOS e Android, além da implantação de novas formas de captura da transação, como o quick response code ( $Q R$ code) e near field communications (NFC). Atualmente, a empresa conta com mais de três mil colaboradores dedicados aos milhões de clientes ativos de diferentes setores da economia.

O sucesso de implantação dos sistemas de pagamento instantâneo dos outros países serve como referência e permite concluir que o Brasil deve aumentar o número de tran- 
sações nos próximos anos. No Brasil, o serviço de pagamento instantâneo pode acelerar a substituição do dinheiro para transações eletrônicas, estimular a inclusão de usuários nos sistemas bancários, gerar oportunidades de negócio e aumentar a competitividade do setor de meios de pagamento (Alfonso, Tombini, \& Zampolli, 2020).

Assim, as seguradoras, que são o setor foco deste trabalho, e outros clientes da adquirente podem usufruir dos benefícios de transferências instantâneas. A inclusão desse serviço no portfólio da empresa pode contribuir no enfrentamento da concorrência e na criação de valor adicional para os clientes, além de potencializar receitas.

O setor de seguros se destaca pela sua relevância econômica, pois se trata de um segmento em crescimento exponencial: segundo dados da CNSEG (2020), o aumento nominal de 2018 para 2019 foi de 8\% com a arrecadação de R 270 bilhões.

Por conseguinte, o setor sente o impacto das novas tecnologias, que mudam, inclusive, a forma como os clientes das seguradoras contratam e usam os serviços: a interface e o contato direto com o consumidor ganham espaço em detrimento da intermediação do corretor (Lyskawa et al., 2019). Além do relacionamento direto, as soluções e os produtos tornaram-se mais simples para facilitar a aquisição e o uso dos diversos tipos de seguros (Lyskawa, Kedra, Klapkiv, \& Klapkiv, 2019), o que também converge com a adesão do pagamento instantâneo que busca facilidade dos interessados, entre outros benefícios.

É nesse contexto que surge a oportunidade de fomento da tecnologia de pagamento instantâneo pela empresa adquirente às seguradoras, considerando que o sistema permite diferentes modelos de uso. A inovação também permite o uso como desembolso de fundos das empresas, governos e instituições, em substituição dos métodos atuais de pagamento, como dinheiro, cheques e transferências identificadas.

\section{DIAGNÓSTICO E ANÁLISE}

\section{Procedimentos adotados no diagnóstico}

Neste estudo, o diagnóstico da oportunidade foi realizado em três etapas. A primeira etapa do diagnóstico foi feita por meio de abordagem quantitativa com o auxílio de um 
questionário on-line na ferramenta Survey Monkey, com perguntas de múltipla escolha e direcionadas para colher a experiência de pagamento dos usuários de serviços de seguros. O envio foi realizado por mídias sociais, como Linkedin, Twitter e Facebook, e coletaram-se dados de uma amostra de 124 respondentes no mês de setembro de 2020.

Observou-se que a amostra contemplou a faixa etária de 27 a 51 anos, considerada economicamente e em idade ativa segundo o Instituto Brasileiro de Geografia e Estatística (IBGE, 2020) e elegível para contratação dos serviços de seguros. Além disso, a faixa etária da amostra inclui o grupo de pessoas com maior propensão à contratação de seguros, segundo levantamento feito pelo Instituto Brasileiro de Opinião Pública e Estatística (Ibope, 2019), encomendado pela Prudential do Brasil, considerando que as pessoas com idade entre 35 a 44 anos são mais propensas aos serviços de seguros, pois, em geral, estão em um período de consolidação financeira e formação familiar.

A segunda etapa do diagnóstico envolveu uma abordagem qualitativa por meio de entrevistas com os gestores de duas seguradoras com o objetivo de garantir a interação direta com o cliente da adquirente, conduzidas a partir de um roteiro com oito perguntas abertas sobre as necessidades do mercado e dos usuários de seguros, a percepção sobre os meios de pagamentos e os benefícios do uso da tecnologia de pagamento instantâneo no setor. As entrevistas ocorreram no mês de outubro de 2020. Na terceira e última etapa do diagnóstico, realizou-se outra pesquisa qualitativa com dois gerentes comerciais da adquirente para identificação dos benefícios da oportunidade de pagamento instantâneo também sob o ponto de vista da empresa adquirente.

\section{Apresentação dos resultados do diagnóstico}

A etapa quantitativa mostrou que o seguro automotivo foi contratado por $83 \%$ da amostra, seguido dos seguros de vida e viagem, com $73 \%$ e $67 \%$, respectivamente. Os dados da pesquisa reforçam o mencionado no levantamento feito pela Superintendência de Seguros Privados (Susep, 2020), que destaca o seguro automotivo como o segundo tipo de seguro mais vendido em 2020 no Brasil, ficando atrás somente do segmento de pessoas pelo quarto ano consecutivo. 
Sobre a intenção de uso dos valores nos finais de semana pelos segurados, visto que se trata de um dos benefícios do pagamento instantâneo, observou-se que 51\% da amostra afirmou ser moderadamente, ou muito, provável o uso dos valores em dias não úteis, o que reforça o pagamento instantâneo como meio de atendimento da necessidade desses usuários.

Nesse sentido, com o objetivo de avaliar se o uso imediato da indenização do seguro poderia ser considerado pouco ou nada provável na população de segurados, procedeu-se à análise estatística. Considerando-se a escala Likert de quatro pontos para essa questão com grau de segurança igual a dois, variabilidade igual a um e precisão igual a 0,33, recomenda-se o tamanho de amostra mínima igual a 37 respondentes (Hair, Babin, Money, \& Samuel, 2005), o que se entende como parâmetro atingido neste diagnóstico. $\mathrm{O}$ resultado do teste de diferença do escore médio da escala $(\mathrm{t}=-7,169, \mathrm{p}$-valor $=0,000)$ indicou que há diferença significativa contrária a um uso pouco ou nada provável sobre utilização imediata da indenização do seguro pelos respondentes. Dada a significância resultante do teste de independência, esse resultado não pode ser considerado acaso.

Tão importante quanto entender as necessidades, intenções de uso e os benefícios valorizados pelos usuários de seguros, a pesquisa quantitativa buscou a relação do pagamento instantâneo com os tipos de seguros, sendo o seguro de viagem o serviço de maior aderência para tecnologia - 79\% das citações. Entende-se que os usuários desse tipo de seguro, em geral, contratam o seguro com poucos dias de antecedência da viagem e que precisam receber os reembolsos e indenizações com urgência na ocorrência de sinistros.

Como citado nos procedimentos do diagnóstico, a segunda etapa contou com abordagem qualitativa por meio de entrevista com dois gestores de seguradoras, o que permitiu o contato direto com o cliente da adquirente e o aprofundamento das suas percepções sobre o pagamento instantâneo nos seus negócios. A primeira entrevista ocorreu com um gestor (entrevistado A) com 35 anos de experiência no setor de seguros, atuando em diversos tipos de seguros massificados, como o automotivo e o residencial, e também nos tipos de seguros corporativos e de riscos empresariais. O entrevistado A trabalha em um grupo de seguros criado em 1984, que atua em diferentes países. No Brasil, a empresa conta com mais de 550 colaboradores distribuídos por nove escritórios em sete estados. 
Segundo o entrevistado A, a necessidade mais latente do consumidor é a postergação e o parcelamento do pagamento da apólice de seguro por meio da emissão e reemissão de boleto. É comum o pedido do consumidor de alteração de boleto, o que pode ser considerado um meio de pagamento seguro, mas também burocrático, principalmente se comparado com outros meios eletrônicos disponíveis.

Ao ser questionado sobre o conhecimento de pagamento instantâneo, o entrevistado A afirmou ter tido contato com o assunto, principalmente em decorrência da mídia relacionada ao cadastro do Pix, e acrescentou:

Tudo que chega para simplificar a venda dos seguros agrega valor para as seguradoras e seus clientes. O pagamento via boleto é um meio arcaico, bem ultrapassado, e precisa ser substituído por outras opções seguras, e o pagamento instantâneo pode ser esta opção. Além disso, a tecnologia de pagamento instantâneo vai de encontro com o investimento de várias seguradoras para tornar a experiência do cliente mais simples e rápida, o que inclui aplicativos de autoatendimento (entrevistado A).

Para o entrevistado A, os benefícios do pagamento instantâneo ficam mais explícitos na necessidade de recebimento de reembolsos e indenizações, visto que após a validação dos documentos, a confirmação do pagamento da apólice pela seguradora para o segurado demorava, na época em questão, de 15 a 30 dias, o que contribui para o entendimento de que o uso do pagamento instantâneo para o desembolso de fundos agrega valor ao setor.

Já a segunda entrevista ocorreu com um gestor (entrevistado B) de uma seguradora vinculada a um banco que atua nos ramos de vida, habitacional, rural e massificados, o que inclui os seguros residenciais, empresariais e de condomínio, com produtos comercializados nas agências do banco e em diferentes canais digitais. Para esse entrevistado, o mercado de seguros não tem ainda uma forma de transferir os valores com segurança e simplicidade para ambos os lados e citou que a sua seguradora usava ordem de serviços no passado, o que permitia o saque em agências de diferentes bancos pelos segurados, mas o modelo tinha um custo elevado e era vulnerável a fraudes. 
É uma vantagem incrível, o pagamento instantâneo é um novo sistema que vai abrir outras possibilidades de serviços e produtos nos mercados, inclusive no setor de seguros. A tecnologia muda toda a cadeia de pagamento, poderá permitir o pagamento de um sinistro na mesma hora para um usuário que tenha um score de risco baixo e um histórico positivo com a seguradora (entrevistado B).

A pesquisa qualitativa foi complementada com a entrevista de dois gerentes comerciais da adquirente na última etapa do diagnóstico. A primeira entrevista ocorreu com um gerente comercial da empresa adquirente (entrevistado C), com experiência de três anos no setor de seguros. O entrevistado C tem histórico no atendimento comercial também em outros setores, o que permite uma análise mais ampla sobre a empresa e sobre o impacto do pagamento instantâneo no negócio.

$\mathrm{O}$ entrevistado $\mathrm{C}$ menciona que há uma demanda das seguradoras por produtos digitais. Geralmente, esse tipo de cliente tem a preocupação de viabilizar uma experiência simples para os usuários, para evitar que os segurados abandonem a compra e procurem a concorrência. A mesma facilidade almejada das seguradoras para os segurados também é exigida da adquirente na implantação e integração. $\mathrm{O}$ entrevistado $\mathrm{C}$ afirma que o "medo" dos clientes é que o desenvolvimento de integração exija um alto investimento ou um prazo muito longo, ou seja, a oferta da adquirente precisa ser simples, via application programming interface (API), com uma documentação de desenvolvimento divulgada, que tenha disponibilidade para testes, de preferência em plataformas de desenvolvimento conhecidas e já utilizadas pelos desenvolvedores e complementa:

Se o processo de implantação for difícil, as seguradoras podem nem querer iniciar o desenvolvimento ou desistirem antes da disponibilização para os seus segurados. Os benefícios de pagamento instantâneo só podem ser identificados pelos usuários se esta for concluída com sucesso (entrevistado C).

Já a quarta entrevista ocorreu com um gerente comercial (entrevistado D) que tem experiência superior a cinco anos no setor de seguros. Ele é também um gestor de pessoas 
e responsável por carteiras de clientes em outros estados e de diferentes setores, com um maior foco em setores com baixa representatividade no uso de cartão como meio de pagamento - como educação e governo, por exemplo, clientes com barreiras para o uso de pagamentos digitais em decorrência da legislação, da sensibilidade de preço e da cultura de uso. O entrevistado D e sua equipe estimulam a criação de valor, posicionando os seus produtos como solução para as "dores" dos seus clientes e acredita que o pagamento instantâneo ajuda a solucionar barreiras não atendidas pelo portfólio atual da empresa:

A empresa possui diferenciais, mas estes não atendem 100\% dos clientes. O pagamento instantâneo permite o retorno em clientes que recusaram a nossa proposta, e mais que isto, viabiliza o atendimento de necessidades ainda não exploradas (entrevistado D).

Particularmente, o diagnóstico revelou a importância do pagamento instantâneo no setor de seguros e reforça a oportunidade de oferta da tecnologia pelas empresas adquirentes, visto que há uma necessidade de recebimento de valores pelo segurado, em casos de reembolso, indenização ou cancelamento, ou seja, uma demanda para o pagamento acelerado, além da demanda de uma inovação pela seguradora para o desembolso de fundos.

\section{SOLUÇÃO ADOTADA}

A solução definida neste trabalho se descreve como "oferta de serviço de pagamento instantâneo para seguradoras, de maneira que esses clientes possam adaptá-los de acordo com suas necessidades, assim como para o desembolso de fundos aos seus segurados, com a opção de tecnologia Pix e das bandeiras de cartão, e uma comunicação fácil e direcionada para o setor e um preço promocional de lançamento". A adoção dessa alternativa converge também para um efetivo mix de marketing, pois trata do conjunto de pontos de interesse dos clientes da empresa, conforme Kotler e Keller (2006): a empresa produz o bem ou serviço (produto), o cliente precisa ser devidamente comunicado (promoção), sendo este distribuído nos canais de venda (praça) e com o valor adequado (preço). 
A inovação permitirá uma nova fonte de receita para a empresa e a fidelização dos seus clientes do setor de seguros. Segundo Tidd, Bessant e Pavit (2008), existem quatro tipos de inovação: produto, posição, processo e paradigma, a solução adotada se relaciona com o primeiro aspecto citado, dado que agrega para o portfólio de produtos da empresa mais uma opção que pode ser explorada no setor de seguros ou em qualquer outro, mas pode ser interpretada também como inovação de posição, já que tem o potencial de melhorar o desempenho competitivo da adquirente e expandir o modelo de negócio atual.

\section{INTERVENÇÃO}

A intervenção foi baseada em plano de ação construído para viabilizar a implantação do pagamento instantâneo na empresa adquirente, em referência ao processo de desenvolvimento de produtos (PDP) apresentado por Echeveste e Ribeiro (2010). Considerando que a execução do projeto estava em andamento até o momento da escrita desse texto, apresentam-se somente as ações realizadas e os seus respectivos detalhes para expor os ganhos com a implantação do serviço. Sendo assim, na sequência, serão apresentadas as fases de pré-desenvolvimento e desenvolvimento da intervenção.

\section{Pré-desenvolvimento}

A fase de pré-desenvolvimento foi composta das seguintes subfases: a) planejamento e b) direcionamento. A subfase de planejamento buscou o diagnóstico da oportunidade no mercado de meios de pagamento, por meio do contato direto com o usuário final, com as seguradoras e com a empresa, com a identificação dos atributos valorizados em toda cadeia. Esse planejamento foi fundamental para que o plano do projeto fosse iniciado e autorizado na adquirente a partir de reuniões de trabalho e apresentações com os tomadores de decisão, e foi importante também para que cada colaborador colocasse suas percepções sobre o projeto.

A ação de análise dos ambientes externo e interno teve por objetivo definir os principais ofensores e itens de impacto do mercado de meios de pagamento, do setor de seguros 
e da própria empresa. Considerando que o assunto de pagamento instantâneo é incipiente, principalmente no setor de seguros, a análise contribuiu para reforçar o desenvolvimento via API, a fim de facilitar a integração e o desenvolvimento sistêmico, o que se torna um requisito no desenvolvimento do projeto.

Quanto à ação de diretrizes de lançamento, o foco foi formalizar um padrão de informações para facilitar o contato com os clientes, com a garantia de transparência e, principalmente, com a redução da complexidade de desenvolvimento para os desenvolvedores.

Nessa etapa do projeto, definiu-se que a abordagem dos clientes para o piloto exigiria a entrega de um kit de desenvolvimento, o que incluiu uma documentação técnica e funcional e um ambiente de testes para simulação de submissão de transações antes da disponibilização em produção para os usuários reais.

A fase de pré-desenvolvimento e suas respectivas ações foram revisadas de forma constante durante o projeto, o que permitiu aos gestores e à equipe alocada no projeto realizar os ajustes necessários nas ações em andamento ou nas ações futuras, além de promover o alinhamento entre as áreas de produtos, finanças e planejamento estratégico, sendo esta última área considerada fundamental para o posicionamento como uma entrega de inovação de pagamento e transformação digital.

\section{Desenvolvimento}

A fase de desenvolvimento na adquirente considerou as subfases: a) projeto; b) recursos humanos; c) comunicação; e d) lançamento do serviço. As ações foram atualizadas a partir dos riscos levantados e foram importantes para viabilizar a execução da implantação do pagamento instantâneo. As subfases foram detalhadas nas próximas seções.

\section{Projeto}

O projeto considerou o desenvolvimento de uma plataforma de pagamento instantâneo por uma adquirente, o que permite explorar diferentes casos de uso para os clientes e 
usuários, mas que no presente trabalho tem o foco no caso de uso de desembolso de fundos das seguradoras para os segurados. Além disso, considerou o desenvolvimento de um protótipo, execução de uma prova de conceito (proof of concept [POC]) com clientes parceiros e o desenvolvimento de processos internos para operação do novo serviço.

Conforme a Figura 1, na jornada do cliente proposta, o portador de cartão e também segurado abre um processo de sinistro ou reembolso para a seguradora, esta que recusa ou aprova a solicitação. Em casos de aprovação, o envio de dados para o crédito é autorizado e direcionado para o Gateway, que atua como empresa intermediária na POC e no projeto de pagamento instantâneo. Essa empresa é responsável por facilitar a integração com as empresas adquirentes e por armazenar os dados transacionais em um local seguro.

Com o pedido de desembolso de fundo da seguradora para a adquirente, são consultados o saldo da seguradora na conta digital e a transitória disponível para consumo da adquirente. Para a implantação do modelo, a seguradora aporta previamente um montante equivalente à demanda projetada de transação em uma conta digital.

Após a validação, a transação é enviada para a bandeira de cartão processar a movimentação do dinheiro em conjunto com o banco emissor. O crédito é disponibilizando em até 30 minutos para o segurado, independentemente de ser dia útil ou horário comercial, todos os dias do ano.

Figura 1 - Jornada do cliente no caso de uso de desembolso de fundos

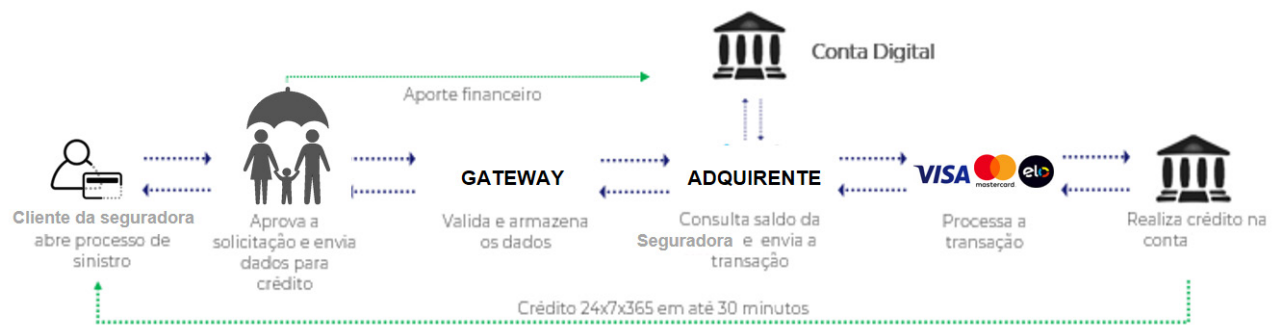

Fonte: Elaborada pelos autores.

Em transações eletrônicas instantâneas, essas operações necessariamente envolvem um cartão de destino para o recebimento dos fundos. Este deve estar previamente cadas- 
trado em um processo conhecido como onboarding, em que o portador inclui os dados do cartão anteriormente e um processo de validação de segurança é executado, permitindo o envio ou o recebimento de valores, conforme exemplo apresentado na Figura 2.

FigURA 2 - Exemplo de experiência de onboarding
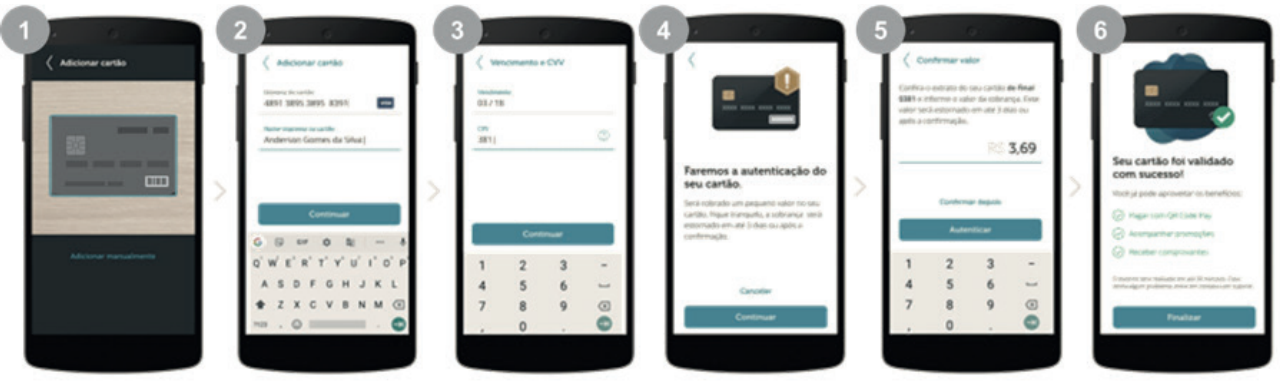

Fonte: Elaborada pelos autores.

Os players são obrigados a realizar consulta em bancos de dados globais a fim de contribuir para prevenção a fraude no país e, após as validações, as transações podem ser negadas. Além da adquirente, as bandeiras de cartão têm limites máximos de quantidade e valor para as transações, com autorização para negar transações na eventualidade de as solicitações ultrapassarem os limites estabelecidos.

Os dados coletados no processo de onboarding precisam ser armazenados em diretório que pode ser do cliente parceiro ou da adquirente, sendo o player definido na negociação o responsável pelo cadastro, validações de prevenção à fraude e atendimento de regras e normas de segurança nacionais e globais.

O projeto teve sua base fundamentada na metodologia ágil, com um squad estruturado e um grupo multifuncional, o que permitiu entregas parciais e a rápida atuação sobre os impeditivos do projeto. Nesse caso, o desafio foi errar no início do projeto, identificar e corrigir rapidamente o erro. Assim como citado por Schwaber (1995), os times de desenvolvimento do projeto tiveram como característica revisões frequentes e colaboração.

A POC contemplou o teste e o uso da plataforma de pagamento instantâneo para viabilizar transações de envio de fundos das seguradoras para os segurados e, para isso, 
foram abordados cinco clientes parceiros, dos quais três avançaram nas negociações jurídicas e comerciais, recebendo kit de desenvolvimento e integração do serviço da adquirente.

Os parceiros que aderiram à POC obrigatoriamente assinaram um contrato de prestação de serviço e sigilo da informação, a fim de mitigar o risco de vazamento de dados. Antes do início dos testes, cada parceiro foi submetido a um processo de aprovação e cadastro. Essa etapa contou com o preenchimento de um formulário de escopo de projeto, validado pelas áreas internas de finanças, prevenção à fraude, segurança da informação e jurídica.

Vale citar que a empresa estava preparada para os dois tipos de pagamento instantâneo: transferências de valores por meio dos sistemas das bandeiras de cartão Visa, Mastercard e Elo ou via Pix, que é o modelo do BCB. O teste inicial foi realizado com a comunicação do serviço por meio das bandeiras de cartão, ou seja, o processamento das transações foi regido a partir das regras dos manuais e publicações das bandeiras de cartão.

A POC contemplou cartões de débito, crédito e pré-pago. Embora a liberação dos tipos de cartão seja uma decisão dos bancos emissores para cada um dos usuários, o teste foi importante para validar a plataforma para todos os tipos de cartão possíveis de transação instantânea.

A integração com os clientes parceiros foi realizada por meio de uma API, o que facilitou o desenvolvimento e a integração à plataforma de pagamento instantâneo. Outro item atendido foi a identificação nos sistemas e relatórios separados das transações de compra atuais da adquirente, ou seja, evitou-se qualquer impacto na operação atual da empresa e a separação também foi mantida no armazenamento das transações realizadas na nova plataforma com a entrega de um repositório segregado.

Para atender à necessidade de desenvolvimento de processos internos específicos para o novo serviço de pagamento instantâneo, um fluxo de cobrança do parceiro foi criado, assumindo como premissa que a movimentação do valor da transferência da seguradora para o segurado seria permitida somente após a transferência de valor suficiente para compensar a demanda transacional de uma conta digital do cliente parceiro para uma conta digital da adquirente, ou seja, antes do processamento da transação de desembolso de fundos. 
Outro processo entregue está relacionado com os casos em que a transação não é autorizada pela bandeira ou emissor do cartão. Nesse caso, o valor da transação não efetivada retorna para conta digital do parceiro. Um fluxo de monitoramento do saldo das contas digitais também foi construído para garantir a disponibilidade de saldo na conta digital do parceiro para consumo e acompanhamento das áreas de tesouraria e finanças.

O processo de cobrança durante a POC e o processo para os clientes que aderirem ao serviço após o projeto seguiram as condições comerciais negociadas entre o parceiro e a adquirente e podem variar conforme a alçada de precificação comercial. Na liquidação das transações de desembolso de fundos, os valores foram debitados da conta liquidante da adquirente e do parceiro, acrescidos da condição comercial, do valor de intercâmbio, que é a remuneração do banco emissor, e somados também à tarifa da bandeira, que é a remuneração da bandeira do cartão, trafegado pelo sistema da Câmera Interbancária de Pagamentos (CIP) de acordo com a regulação vigente.

A disponibilização do serviço de pagamento instantâneo pelo projeto durante a POC beneficiou os portadores de cartão com a experiência de receber rapidamente valores originados das seguradoras, o que é uma contribuição da empresa também para a sociedade ao oferecer uma alternativa para o papel-moeda, para o cheque, para a transferência eletrônica de fundos (TED), documento de ordem de crédito (DOC) e ordens de pagamento. A iniciativa contribui para inclusão de pessoas não bancarizadas nos meios de pagamento eletrônico por meio da aceitação de cartões pré-pagos.

Para a adquirente, o projeto também contribuiu para um posicionamento estratégico com a imagem de inovação e transformação digital e como uma nova fonte de receita, com menor custo sistêmico que uma transação de compra, além de atender à necessidade das seguradoras de pagamento mais rapidamente do que um sinistro, por exemplo.

\section{Recursos humanos}

A subfase de recursos humanos foi composta por duas ações: a) organização de equipes multifuncionais; e b) treinamento, importante para permitir a implantação do pagamento instantâneo, pois nessa fase do projeto os gestores e executivos da empresa participaram 
das definições do novo serviço e identificaram o valor do projeto como uma etapa importante para a transformação digital e a busca de inovação.

A ação de organização de equipes multifuncionais possibilitou a formação de um time de projeto heterogêneo, unindo capacidades e conhecimentos diferentes e organizando-os para o objetivo comum de implantação do projeto. Na iniciativa, foram alocados recursos das áreas de produtos, tecnologia, segurança da informação, marketing e comercial.

Nessa etapa, uma área para gestão da plataforma de pagamento instantâneo foi criada para garantir a continuidade do desenvolvimento e principalmente a visão de negócio alinhada às diretrizes e metas de planejamento estratégico, além de ser responsável por ações de fomento e busca de clientes parceiros junto com os times comerciais, o que é importante para rentabilizar os investimentos e diminuir a incerteza da nova tecnologia no mercado. Desse modo, colaboradores foram alocados e movimentados para o projeto e novas pessoas passaram a fazer parte do quadro de funcionários com o objetivo de complementar as equipes com conhecimentos externos e específicos de pagamento instantâneo ou de segurança.

A ação de treinamento da área de atendimento ocorreu em paralelo com o desenvolvimento do projeto e foi desenvolvida pelas equipes que construíram os fluxos e participaram das decisões. Isso contribuiu para maior qualidade e quantidade de detalhes nos materiais de suporte. A aplicação para equipes foi adaptada conforme o público, assim, os operadores das centrais de atendimento não necessariamente receberam o mesmo procedimento que os gerentes comerciais.

A universidade corporativa da empresa também foi envolvida e influenciou as metodologias de aprendizagem dos colaboradores, além de disponibilizar diretórios para os materiais de suporte e consulta posterior.

\section{Comunicação}

A subfase de comunicação foi iniciada praticamente no início do projeto, mas intensificou-se após a etapa de desenvolvimento e durante a abordagem dos clientes parceiros 
para POC, dividida em duas frentes de atuação. Contemplou a comunicação interna para os colaboradores e áreas de atendimento, e a comunicação externa direcionada para os clientes.

$\mathrm{Na}$ comunicação interna, além dos treinamentos descritos na subfase de recursos humanos, foi realizada a divulgação na intranet da empresa e o maior alcance atingido na apresentação dos fóruns e comitês da empresa quando os materiais foram apresentados, dando visibilidade dos detalhes de desenvolvimento, complexidade, impeditivos e próximas etapas de execução. Essas ações foram importantes para garantir a agilidade na tomada de decisão e a correção de estratégias.

A comunicação externa foi direcionada para as seguradoras com o objetivo de valorizar os benefícios de pagamento instantâneo e reduzir a percepção de complexidade da integração por meio de apresentações preparadas para suportar as reuniões de trabalho dos gerentes comerciais e fomentar a adesão dos clientes. Dos cinco clientes abordados com os materiais, três clientes seguiram o desenvolvimento, então a comunicação pode ser considerada efetiva e relevante. Os materiais foram preparados com o auxílio da área de marketing e contiveram principalmente a simplificação dos fluxos técnicos.

\section{Lançamento do serviço}

O lançamento do serviço de pagamento instantâneo pela adquirente ocorreu na segunda quinzena de março de 2021, com a união de áreas como produtos, marketing e comercial e a divulgação na mídia da parceria com uma seguradora global e centenária, presente no mercado brasileiro desde 1984, em outros 214 países e territórios, com aproximadamente 55 mil funcionários. A seguradora atende a pessoas físicas e jurídicas - de pequenas empresas a multinacionais, com uma ampla quantidade de produtos e serviços de seguro.

O início das operações do novo serviço permitiu a transferência de valores entre a seguradora e seus segurados em tempo real e, conforme identificado na etapa de diagnóstico, a seguradora optou por realizar esse tipo de pagamento para atender à necessidade do usuário de solicitações de reembolso para as coberturas de atraso de voo e despesas farmacêuticas conforme estabelecido pelo respectivo contrato de seguro viagem. 
Com essa solução implantada, o tempo para o segurado receber a indenização após a aprovação da seguradora é reduzido de cinco dias úteis para 30 minutos aos portadores autorizados pelos bancos emissores na modalidade de pagamento instantâneo. Após a aprovação da solicitação, o usuário recebe um e-mail com a confirmação de que o seu pedido de reembolso foi aprovado e o link para inserir os dados do cartão para que seja realizado o crédito no valor devido.

\section{AVALIAÇÃO}

A avaliação da implantação do pagamento instantâneo foi realizada a partir de três etapas de coleta de dados. A primeira etapa foi realizada por meio da análise documental de uma entrevista da agência de notícias Reuters no lançamento da tecnologia (Alves, 2021, 15 de março) com o diretor responsável pelo departamento de Vida, Previdência e Capitalização no Brasil, denominado neste trabalho de entrevistado E. Para ele, a iniciativa posiciona a seguradora parceira da adquirente no lançamento do pagamento instantâneo entre as mais ágeis no atendimento aos segurados. A solução reduz o tempo para o segurado receber as indenizações de cinco dias úteis a 30 minutos. Em paralelo, a tecnologia reduz em, aproximadamente, $6 \%$ os custos transacionais de pagamentos, na comparação com uma transação de compra de crédito à vista com a referência de taxa de $3,1 \%$, em razão do menor custo de transação.

O entrevistado E citou também que o objetivo é expandir a solução para outras 34 coberturas reembolsáveis do seguro viagem, após o lançamento do pagamento instantâneo na contratação do serviço e no atendimento inicial das solicitações de reembolso para as coberturas de atraso de voo e despesas farmacêuticas, além da expansão para outras linhas de negócios aderentes ao serviço instantâneo. A inovação converge com a estratégia das seguradoras de fomentar os canais eletrônicos, simplificar a relação com os usuários e identificar novos meios de fidelizar os segurados.

A segunda e a terceira etapa envolveram entrevistas com dois representantes da adquirente, com o foco de coletar a percepção da adquirente. A primeira entrevista ocorreu com um gerente comercial da adquirente, detentor de uma carteira de clientes 
concentrada no setor de seguros e em segmentos com baixa cultura de uso de pagamento eletrônico e experiência de dois anos no setor de meios de pagamento e denominado entrevistado F.

Segundo o entrevistado F, a avaliação da implantação do projeto de desembolso de fundos com as seguradoras envolveu três desafios: a concorrência interna com outros projetos da adquirente, inclusive a necessidade de priorização de iniciativas, como o desenvolvimento do Pix. O segundo desafio mencionado pelo entrevistado F está relacionado com a novidade do assunto, posicionado como uma inovação para os setores de meios de pagamento e de seguros. A implantação do projeto exigiu o esforço inicial para definir os itens necessários, sendo fundamental a cooperação entre as empresas para indicar intermediários e apreender e usar os dados da POC na tomada de decisão. O último desafio, destacado como um dos mais relevantes para o entrevistado F, foi o impacto causado pela pandemia da Covid-19. A pandemia impactou diretamente o segmento de turismo e viagens, definido pelas seguradoras como inicial para a implantação do pagamento instantâneo.

$\mathrm{Na}$ avaliação do entrevistado F, a implantação do pagamento instantâneo e o uso como desembolso de fundos atendem à necessidade dos usuários das seguradoras de receber de forma rápida o que for devido, mesmo que seja em dia não útil ou fora do horário comercial, e facilitam a experiência de uso dos serviços de seguros. O entrevistado F mencionou ainda a maior exposição do assunto de pagamento instantâneo nos últimos meses, principalmente relacionado ao modelo proposto pelo BCB, o Pix. Além da comunicação direcionada para as seguradoras realizada pela adquirente, essas ações contribuíram para o aumento da adesão de seus clientes na oferta do serviço da adquirente e para o fomento em outros clientes.

A entrevista seguinte ocorreu com um integrante do projeto (entrevistado G) com experiência superior a dez anos no setor de meios de pagamento, com atuação em departamentos estratégicos da empresa. O entrevistado $\mathrm{G}$ também conseguiu avaliar três desafios com o projeto de pagamento instantâneo. O primeiro está vinculado ao conhecimento da tecnologia. Segundo o entrevistado G, os colaboradores precisaram pensar diferente e usar a experiência da adquirência para construir o novo projeto. 
Na avaliação do entrevistado G, o outro desafio foi a mobilização das pessoas. A construção da plataforma instantânea e a execução da POC exigiram um grupo multidisciplinar para conduzir a iniciativa. Também foram necessários o recrutamento externo e a contração de equipes terceiras. As áreas da empresa precisaram organizar o escopo de trabalho para ceder recursos ao projeto, que foi estruturado sob metodologia ágil, obrigando a alocação total da maior parte dos colaboradores. O terceiro desafio mencionado pelo entrevistado $G$ foi o prazo reduzido para desenvolver o projeto e a POC, a fim de atender à necessidade do mercado e principalmente para garantir o posicionamento de ser a primeira adquirente com a inovação.

O volume de transações de desembolso de fundos não foi divulgado, pois é uma informação confidencial da empresa, porém é de conhecimento que o pagamento instantâneo contribui para o resultado da adquirente, pois é um resultado incremental às transações de compra. As ações implementadas no ano de 2020 e no início do ano 2021 foram essenciais para viabilizar a implantação da plataforma de transferência on-line, a transação de pagamento instantâneo e a execução da POC de desembolso de fundos com a seguradora.

\section{CONSIDERAÇÕES FINAIS}

A experiência da implantação do pagamento instantâneo e do uso para o desembolso de fundos das seguradoras para os segurados com o método de design science research sob o modelo de problem-solving proposto por Van Aken, Berends e Van der Bij (2012) e detalhado em Marcondes, Miguel, Franklin e Perez (2017) é de grande relevância para o aprendizado profissional de quem está interessado em implementar soluções em organizações.

De fato, desde a execução do plano de ações proposto até a etapa de intervenção, os conceitos metodológicos foram fundamentais para conduzir as atividades, além de permitir a reflexão sobre pontos de melhoria. Na fase de pré-desenvolvimento, a contribuição do presente trabalho se deu na proposição e execução de um diagnóstico quantitativo e qualitativo da oportunidade a partir de percepção dos usuários do serviço, das segura- 
doras e da empresa, a adquirente desenvolvedora do projeto. A subfase de planejamento foi essencial para o alinhamento com os tomadores de decisão e para que o projeto fosse iniciado na adquirente. Já as análises dos ambientes externo e interno permitiram a identificação de diretrizes e o alinhamento com a liderança da empresa e contribuíram para reforçar o desenvolvimento e a integração do serviço via API, a fim de facilitar a integração com os clientes. Nesse momento do projeto, definiu-se também que a abordagem dos clientes para o piloto exigiria a entrega de uma documentação e um ambiente de testes para simulação de submissão de transações antes da disponibilização em produção para os usuários reais.

$\mathrm{Na}$ fase seguinte de desenvolvimento, a contribuição na subfase de projeto ocorreu na proposição de uma POC com a seguradora, o que certificou o correto funcionamento do pagamento instantâneo fora do sistema da adquirente integrado com o cliente e com transações de pagamento instantâneo reais no ambiente de produção. Na subfase de recursos humanos, o impacto se deu no alinhamento e na identificação do projeto como uma etapa importante para a transformação digital e na busca de inovação pelos gestores da adquirente. A própria ação de organização de equipes multifuncionais estimulou o aproveitamento do capital intelectual dos colaboradores atuais e a sinergia entre diferentes departamentos da empresa.

A subfase de comunicação foi dividida em duas frentes de atuação: a comunicação interna para os colaboradores e áreas de atendimento e a comunicação externa direcionada aos clientes, sendo esta última frente a principal contribuição da subfase, por meio das apresentações preparadas para suportar as reuniões comerciais e fomentar a adesão dos clientes.

Por fim, na fase de desenvolvimento, o próprio lançamento do serviço de pagamento instantâneo pela adquirente permitiu a transferência de valores entre a seguradora e seus segurados em tempo real, inicialmente na contratação de seguro viagem.

Já na última fase, relacionada com o pós-desenvolvimento, a proposição do trabalho foi a avaliação e o registro dos dados, por meio de indicadores de acompanhamento do projeto. Isso permitiu ações de ajuste na operação a partir de dados quantitativos e não somente na experiência dos envolvidos. A ação permitiu avaliar a efetividade da implan- 
tação do pagamento instantâneo, não apenas em termos de volume de transações, mas a partir de outros indicadores definidos como importantes para a empresa, como tempo de disponibilização dos valores para os usuários em até 30 minutos, disponibilidade de solicitações realizadas em dias não úteis e fora dos horários comerciais, sendo esses também os principais benefícios identificados pelas seguradoras e por seus usuários.

Em resumo, a disponibilização do serviço de pagamento instantâneo para o desembolso de fundos das seguradoras beneficiou os portadores de cartão com a experiência de receber rapidamente os valores originados das seguradoras, 24 horas por dia, nos sete dias da semana e em todos os 365 dias do ano, o que reflete uma contribuição da solução para a sociedade.

Para a adquirente, o projeto contribuiu para o posicionamento estratégico e para a imagem de inovação da empresa. O fato de ser um dos primeiros players a lançar o pagamento instantâneo permite que a solução associe a marca da adquirente com a nova tecnologia pelos clientes do serviço. O serviço de pagamento instantâneo contribui também para o resultado da empresa, já que é incremental ao volume atual das transações de compra.

A solução implementada endereça a necessidade das seguradoras de pagamento rápido de um sinistro, por exemplo e, em paralelo, atende a outros setores. Após o lançamento do serviço e a respectiva comunicação, clientes de outros segmentos procuraram a adquirente em busca de informações comerciais e técnicas e dois estão em andamento da negociação comercial, o que reforça a aderência do pagamento instantâneo também em outros setores e possibilita novas oportunidades de negócio no curto e médio prazo para a empresa.

Em relação à complexidade da implantação da tecnologia, o time comercial precisou superar a rejeição inicial da nova tecnologia e expor os benefícios do pagamento instantâneo para garantir a adesão dos clientes e, para isso, foram realizadas reuniões e apresentações dos fluxos de integração e desenvolvimento da API do serviço. A interação com as bandeiras de cartão, principalmente com as empresas globais, para garantir a adaptação dos serviços à realidade brasileira, também contribuiu para a maior complexidade do projeto. Por fim, o outro ponto de complexidade foi a necessidade de alinhamento com 
os bancos emissores e com o BCB, a fim de deixar explícita a segurança e a qualidade adquiridas durante todo o processo de desenvolvimento da inovação e garantir a permissão para a comercialização do serviço aos portadores previamente autorizados.

Como sugestão aos interessados em viabilizar a implantação do pagamento instantâneo, recomenda-se o alinhamento com a liderança da empresa. Deve-se entender o modelo de negócio vigente e identificar o que a empresa possui, o que precisa desenvolver, os recursos e os conhecimentos necessários. Uma análise do mercado e da concorrência deve ser construída para que fiquem claros os pontos de diferenciação que podem ser explorados com os clientes. Na sequência, é fundamental o desenvolvimento de uma plataforma e/ou sistema dedicado para o serviço, o que inclui o processo de onboarding dos dados coletados na entrada dos usuários e o armazenamento em um diretório, validações de segurança, principalmente para manter a gestão da nova tecnologia independente das transações de compra e de outros negócios que a empresa já tenha. A integração com os clientes parceiros deverá ser por meio de uma API, para facilitar a adesão à plataforma de pagamento instantâneo. É recomendada execução da POC antes da expansão do serviço, a fim de validar o fluxo da transação e a integração com o cliente, quando é possível corrigir erros e identificar as pendências do projeto de modo mais explícito. O teste permite ainda a validação do cumprimento das regras dos manuais e publicações das bandeiras de cartão, além das normas dos emissores de cartão.

Como contribuições adicionais, espera-se que o presente trabalho agregue conhecimento para o entendimento da indústria de meios de pagamento, do setor de adquirência e do assunto incipiente de pagamento instantâneo e estimule também outros profissionais a dividir os desafios de diferentes mercados.

\section{REFERÊNCIAS}

Alfonso, V., Tombini, A., \& Zampolli, F. (2020, December 7). Retail payments in Latin America and the Caribbean: Present and future. BIS Quarterly Review, 71-87.

Alves, A. (2021, 15 de março). Zurich e Visa lançam reembolso instantâneo de seguro no Brasil. UOL. Recuperado de https://www.uol.com.br/tilt/noticias/reuters/2021/03/15/zurich-e-visa-lancam-reembolso-instantaneo-de-seguro-no-brasil.htm. 
Associação Brasileira das Empresas de Cartões de Crédito e Serviços (Abecs). (2021). Balanço 2020, Associação Brasileira das empresas de cartões de crédito e serviços. Recuperado de https://api.abecs.org.br/wp-content/uploads/2021/02/Balan\%C3\%A7o-do-Setor-4\%C2\%BA-Trimestre-de-2020-Apresenta\%C3\%A7\%C3\%A3o.pdf.

Banco Central do Brasil (BCB). (2020). Estudos especiais. Recuperado de https://www.bcb. gov.br/publicacoes/estudosespeciais.

Banco Mundial. (2017). The Global Findex Database 2017. Recuperado de https://globalfindex.worldbank.org/.

Braido G., Klein A., \& Papaleo G. (2021). Facilitators and barriers faced by mobile payment fintechs in the Brazilian context. Brazilian Business Review, 18(1), 22-44. doi:10.15728/ bbr.2021.18.1.2

Campos, R., Neto (2021). Meios eletrônicos de pagamentos e agenda BC. Congresso de Meios Eletrônicos de Pagamentos (CMEP) 14, [s. 1.] [on-line].

Cielo. (2021). Relações com investidores, central de resultado. Recuperado de https://ri.cielo. com.br/informacoes-financeiras/central-de-resultados/.

Conductor. (2020). Pagamentos instantâneos pelo mundo: como outros 5 países adotaram modelos como o Pix. Recuperado de https://conductor.com.br/pagamentos-instantaneos/\#: : text=Por\%20isso\%2C\%20bancos\%2C\%20fintechs\%2C,sistemas\%20de\%20pagamentos\%20instant\%C3\%A2neos\%20consolidados.

Confederação Nacional das Empresas de Seguros Gerais, Previdência Privada e Visa, Saúde Suplementar e Capitalização (CNSEG). (2020). Relatório anual de atividades 2018. Recuperado de https://cnseg.org.br/publicacoes/relatorio-anual-de-atividades-2018.html.

Demirgüç-Kunt, A., Klapper, L., Singer, D., Ansar, S., \& Hess, J. (2018). The Global Findex Database 2017: Measuring financial inclusion and the Fintech Revolution. Washington, DC: World Bank.

Echeveste, M. E., \& Ribeiro, J. L. D. (2010). Diagnóstico e intervenção em empresas médias: Uma proposta de (re)organização das atividades do processo de desenvolvimento de produtos. Produção, 20(3), 378-391.

Fan, L., Zhang, X., Rai, L. \& Du, Y. (2021). Mobile payment: The next frontier of payment systems? An empirical study based on push-pull-mooring framework. Journal of Theoretical \& Applied Electronic Commerce Research, 16(2), 155-169. 
Hair, J. F., Jr., Babin, B., Money, A. H., \& Samuel, P. (2005). Fundamentos de métodos de pesquisa em administração. Porto Alegre: Bookman.

Instituto Brasileiro de Geografia e Estatística (IBGE). (2020). Estatística de Gênero. Recuperado de https://www.ibge.gov.br/apps/snig/v1/index.html?loc=P12,P27,P16,P13,P29,P23,P53, P32,P52,P21,P51,P50,P31,P15,P25,P41,P26,P22,P24,P43,P33,P11,P14,P42,P35,P28, $\mathrm{P} 17 \& \mathrm{cat}=-1,-2,-3,128 \&$ ind $=4727$.

Instituto Brasileiro de Opinião Pública e Estatística (Ibope). (2019, 2 de setembro). Apenas 15\% dos brasileiros têm seguro de vida. Recuperado de https://www.prudentialdobrasil. com.br/noticias/imprensa/pressreleases/pesquisa_ibope

Jaworski, B. J., \& Kohli, A. (1993). Market orientation: Antecedents and consequences. Journal of Marketing, 57. 53-70.

Kotler, P., \& Keller, K. L. (2006). Administração de marketing (12a. ed.). São Paulo: Pearson Prentice Hall.

Lima, A. C. (2016). Análise prospectiva da indústria bancária no Brasil: Regulação, concentração e tecnologia. Revista de Administração Contemporânea, 20(5), 546-567. doi:10.1590/ 1982-7849rac2016150053

Lyskawa, K., Kedra, A., Klapkiv, L., \& Klapkiv, J. (2019). Digitalization in insurance companies. International Scientific Conference Contemporary Issues in Business, Management and Economics Engeneering, Vilnius, Lithuania.

Marcondes, R. C., Miguel, L. A. P., Franklin, M. A., \& Perez, G. (2017). Metodologia para elaboração de trabalhos práticos e aplicados: Administração e contabilidade. São Paulo: Editora Mackenzie.

Oxford Analytica (2020). Pix raises competition and regulatory issues in Brazil. Expert Briefings. doi:10.1108/OXAN-ES257615

Schwaber, K. (1995). Scrum development process. Recuperado de http://jeffsutherland.com/ oopsla/schwapub.pdf.

Sgarioni, M. (2021, 17 de fevereiro). Pix ultrapassa 159 milhões de chaves de acesso cadastradas. Mobile Time.

Stankic, R., Aleksic, J. S., \& Jakovljevic, A. (2019). Digital transformation trends in payment systems. New Economist/Novi Ekonomist, (26), 34-41. doi:10.7251/NOE1926034S 
Superintendência de Seguros Privados (Susep). (2020). Relatório Susep de análise e acompanhamento dos mercados supervisionados. Recuperado de http://www2.susep.gov.br/menuestatistica/SES/principal.aspx.

Tidd, J., Bessant, J., \& Pavitt, K. (2008). Gestão da inovação (3a. ed.). São Paulo: Artmed.

Toucinho F. A. (2020). How payments drive digital transformation. Journal of Payments Strategy \& Systems, 14(1), 10-13.

Van Aken, J. E., Berends, H., \& Van der Bij, H. (2012). Problem solving in organizations (2nd. ed.). Cambridge: University Press Cambridge. 